\title{
La internacionalización de los medios de comunicación chinos: un estudio de la producción periodística en CGTN AFRICA
}

\section{The internationalization of Chinese media: a production study of CGTN Africa}

\author{
AUTOR: \\ Dani Madrid-Morales \\ Departamento de Comunicación y Medios \\ Universidad de la Ciudad de Hong Kong
}

\section{RESUMIEN}

Desde mediados de los años 2000, los medios de comunicación estatales chinos han emprendido un proceso de internacionalización con el objetivo de incrementar la capacidad de Pequín de influir sobre la opinión pública global. África, en donde los principales medios chinos (Xinhua, CRI, China Daily...) han establecido sedes regionales, es el continente en donde este proceso se hace más visible. Uno de estos medios es CGTN Africa, centro de producción y difusión de la Televisión Central de China (CCTV) en Nairobi (Kenia). Este artículo usa 36 entrevistas en profundidad con trabajadores y extrabajadores de CGTN Africa para dar respuesta a tres cuestiones: cuál es el proceso de producción periodística de la cadena, cuáles son los valores-noticia imperantes en la redacción y qué impacto tienen sus emisiones sobre los profesionales de la comunicación en el continente. El análisis de las entrevistas revela, por un lado, una permanente negociación entre estructura y agencia, que da lugar a

\section{ABSTRACT}

Since the mid-2000s, China's State-owned media have engaged in a process of internationalization that has the objective of increasing Beijing's ability to sway global public opinion. Africa, where some of the most influential Chinese media (Xinhua, CRI, China Daily...) have set up regional headquarters, is the continent where this process is more clearly seen. One of these news organizations is CGTN Africa, China's Central Television's (CCTV) production and broadcasting centre in Nairobi (Kenya). This paper uses 36 in-depth interviews with current and former CGTN Africa employees to answer three questions: what is the news production process like at the station; what are the news values most commonly used in the newsroom; and, what impact does CGTN Africa have on media professionals in the continent. The analysis of interviews reveals, on the one side, a constant negotiation between agency and structure, which leads to a hybrid news product that could be labelled, paraphrasing other similar 
DANI MADRID-MORALES La internacionalización de los medios de comunicación chinos: un estudio de la producción periodística en CGTN AFRICA.

un producto informativo híbrido, algo que se podría llamar, parafraseando usos similares, "noticias sobre África con características chinas". Por el otro, el articulo pone de relieve la importancia de prestar más atención desde el ámbito académico a los procesos comunicativos que ocurren entre países y regiones del Sur Global.

Palabras Clave África; audiencia; CGTN; China; internacionalización; periodismo uses, "African news with Chinese characteristics." On the other hand, this paper calls for more academic research on communication processes between countries and regions in the Global South.

Keywords Africa; audience; CGTN; China; internationalization; journalism

\section{INTRODUCCIÓN}

A mediados de enero de 2017, la sede africana de la Televisión Central de China (CCTV) celebró su quinto aniversario. Fue una celebración simple en donde la cadena mostró su gratitud a algunos de los 110 profesionales que trabajan en la redacción desde sus inicios. Hubo pastel y vino espumoso, recuerdos de los primeros días y anécdotas, muchas anécdotas... También hubo discursos, incluido el del editor jefe, Pang Xinhua, quien recordó a los presentes, sobre todo periodistas, pero también alguna que otra autoridad invitada, cuál debería ser el principal objetivo de la cadena en África: "Debemos tener nuestro propio punto de vista. No podemos simplemente repetir lo que está pasando o informar igual que lo hacen los medios occidentales. Si simplemente repetimos todo el tiempo, entonces no tiene ningún sentido que estemos aquí. Así que, recordad: ¡debemos mostrar la diferencia!" (CGTN Africa, 2017).

Cuando se fundó en 2012, CCTV Africa, que recientemente ha sido rebautizada como CGTN Africa (del inglés China Global Television Network) dentro de un proceso global de renovación de la marca, se convirtió en el primer centro de producción y emisión de la televisión estatal china en el extranjero (Jacobs, 2012). En cinco años, CGTN Africa ha establecido una de las redes de corresponsales de televisión más extensa en el continente, ha producido más de 3.000 horas de contenido exclusivo sobre África, y ha llegado a acuerdos con múltiples cadenas estatales africanas para poder redistribuir su señal a través de ellos (Mlilo, 2015). CGTN Africa es una de las caras más visibles de la estrategia de internacionalización de los medios de comunicación chinos impulsada por el gobierno. Junto a CGTN Africa y su emisora madre, CGTN, forman parte de esta estrategia global medios como el periódico en inglés China Daily, que cuenta con ediciones específicas para cuatro continentes (Europa, América, Asia y África), la cadena de radio China Radio International (CRI), que emite en más de sesenta idiomas, y la agencia de noticias Xinhua.

La dimensión internacional de los medios de comunicación estatales chinos ha existido desde los años cincuenta, cuando la radio -entonces conocida como Radio Pekín- y las revistas fueron usadas por el régimen maoísta en la batalla ideológica que se libró contra los países capitalistas primero, y más adelante, también contra la Unión Soviética (USIA, 1973; Wekesa, 2017). A diferencia de los años cincuenta y sesenta (Üngör, 2009), el actual proceso de internacionalización cuenta con un sólido respaldo financiero. Según estimaciones aparecidas en 
la prensa -no existen datos oficiales- en 2009, el gobierno chino destinó 45.000 millones de yuanes a la expansión internacional de los medios de propiedad estatal (Barboza, 2009; A. Chen \& Wu, 2009) como Xinhua, CRI o CCTV. En tanto que potencia emergente con aspiraciones de potencia global, China ha apostado por los medios de comunicación como herramienta que pueda ayudar en dicha transición. La presuposición es que como más internacionales sean sus medios de comunicación, mayor será la capacidad de Pequín de contribuir a la formación de narrativas informativas globales favorables a sus intereses en política exterior (Shambaugh, 2013).

Dentro del portfolio de medios chinos en proceso de internacionalización, CGTN Africa, el objeto de estudio de este artículo, presenta uno de los casos más interesantes. El canal opera en un continente, África, que ha estado tradicionalmente en la periferia del sistema comunicativo global (Paterson, 2013); está gestionado por China, un país que es constantemente vilificado por su restrictiva regulación de la libertad de prensa (Ollé, 2017); dispone de instalaciones con la última tecnología y ofrece una cobertura regular en profundidad sin precedentes de la actualidad africana (Marsh, 2016); da empleo a un grupo multinacional de periodistas con perfiles profesionales heterogéneos (Lefkowitz, 2017); y está rodeado de un hermético secretismo acerca de su audiencia y capacidad de influencia (Gorfinkel, Joffe, Van Staden, \& Wu, 2014).

En este artículo se pretende dar respuesta a tres cuestiones ligadas a la práctica periodística. Primero se plantean dos cuestiones: ¿cómo se estructura la redacción de CGTN África y cuáles son los valores noticia imperantes en la redacción? Habiendo dado respuesta a estas dos cuestiones, el artículo pasa a examinar cuáles son las posibles consecuencias para la actividad periodística en África que se devengan de la expansión de los medios de comunicación chinos en el continente. Para ello, se hace uso de 36 entrevistas en profundidad con trabajadores y extrabajadores de CGTN Africa. El artículo comienza con una visión global del proceso de internacionalización de los medios de comunicación chinos, con especial énfasis en el continente africano. A continuación, se presentan los principales resultados del análisis de las entrevistas en profundidad, seguidos de una discusión sobre el encaje de este estudio en la literatura existente acerca de la internacionalización de los medios de comunicación chinos a escala global.

\section{La internacionalización de los medios en China}

El uso de los medios de comunicación por parte de los estados para influir sobre audiencias en otros países es una práctica con más de un siglo de historia. A finales del siglo XIX, el medio para hacerlo era la prensa; en los años veinte del siglo pasado, la radio logró ser tan importante como los periódicos; $y$, desde finales de los años ochenta, la televisión se ha convertido en el medio más prevalente, con permiso de internet. Como recuerda Culbert (2010), el contenido dirigido a audiencias en el extranjero fue denominado, sin complejos, "propaganda" durante la mayor parte del siglo XX:

Durante un tiempo, la palabra tuvo un significado honorable, en el sentido de 'propagar', pero la Primera Guerra Mundial cambió su significado para siempre. En el imaginario público, pasó a ser sinónimo de "mentiras", particularmente mentiras contadas por el gobierno (p. 421).

La circulación de propaganda fue en aumento durante y después de la Segunda Guerra Mundial, "durante la cual la radiodifusión internacional fue usada para generar actitudes positivas hacia ideologías rivales" (Gilboa, 2008, p. 59). China fue especialmente activa durante estos 
años. Las emisiones chinas de radio en japonés e inglés empezaron, respectivamente, en 1941 y 1947 (W. H. Chang, 1989). Hoy en día, el uso del término "propaganda" ha caído en desuso (T.K. Chang \& Lin, 2014) y ha pasado a ser sustituido por el de diplomacia pública, un término que agrupa el conjunto de acciones que los gobiernos toman con el objetivo de "informar, influir e interactuar con ciudadanos de otros países para conseguir apoyos a sus intereses nacionales" (Snow, 2009, p. 6). La línea que separa la "vieja" propaganda de la "nueva" diplomacia pública es fina. Un cambio relevante es la multiplicidad de canales a través de los cuales los gobiernos tratan de conectar con públicos en otros países. Cull (2008) divide las acciones de los gobiernos en cinco categorías: escucha, abogacía, diplomacia cultural, diplomacia de intercambios y radiotelevisión internacional. Durante los años cincuenta y sesenta, China usó con destreza la diplomacia cultural y de intercambios (Passin, 1962) y puso en marcha un ambicioso programa de radiodifusión internacional (Üngör, 2009) que sigue en pie hoy en día tras haberse expandido sustancialmente en los años noventa, cuando la televisión se incorporó a la cartera de medios.

Estrenada en 1992, CCTV-4, una emisora en chino dirigida a las comunidades de ultramar, fue la primera cadena de televisión de China para consumo extranjero. La programación regular en lenguas extranjeras comenzó en septiembre de 2000 con la creación de CCTV-9, un canal en inglés que ofrecía una mezcla de documentales, espectáculos culturales y noticias. Según documento oficiales, la puesta en marcha de la cadena respondía a cuatro objetivos: "promover la excelencia de la cultura china, comunicar los logros de la construcción socialista china, presentar los principios y posiciones de China y establecer una buena imagen de la China socialista" (Zhao, 2008, p. 61). Los primeros años del canal en inglés de la CCTV (del inglés, China Central Television) han sido descritos como "aburridos, propagandísticos y poco profesionales" (Jirik, 2010, p. 149). A partir de 2004 se introdujeron cambios para convertir el canal en uno de orientación "global". El eslogan pasó de "Your Window on China", es decir "Su Ventana a China", a "Your Window on China and the World", "Su Ventana a China y al Mundo (Zhao, 2008, p. 53).

La internacionalización de CCTV entró en una tercera etapa en octubre de 2004 con la creación de un canal en francés y español (CCTV-E \& F). En un primer momento, el contenido en los dos idiomas alternaba en bloques de ocho horas, e iba dirigido sobre todo al público latinoamericano y africano (Madrid-Morales, 2015, p. 201). En 2007 los dos canales se separaron, dando lugar a CCTV-E y CCTV-F. La expansión global concluyó en 2009 con la suma de un canal árabe en julio y otro ruso en septiembre. En poco menos de una década, CCTV pasó de tener un único canal en un idioma extranjero a tener cinco, convirtiendo a China en el único país con canales de televisión las 24 horas en los seis idiomas oficiales de las Naciones Unidas. Desde entonces, las emisiones internacionales han pasado por múltiples procesos de renovación. En 2010, CCTV-9 pasó a ser CCTV-News, un canal de 24 horas de noticias en inglés. Y, en diciembre de 2016, Pequín anunció un cambio de nombre para toda su red de cadenas globales (AP, 2016), abandonando las siglas CCTV y adoptando las de CGTN (China Global Television Network o Red Global de Televisión de China).

Además de televisión, el proceso de internacionalización de los medios chinos también incluye, entre otros, la expansión de emisiones de radio a través de la Radio Internacional de China o CRI (C. C. Chen, Colapinto, \& Luo, 2010), la publicación del diario China Daily, la apertura 
DANI MADRID-MORALES La internacionalización de los medios de comunicación chinos: un estudio de la producción periodística en CGTN AFRICA.

de decenas de corresponsalías de la agencia de noticias oficial Xinhua (Xin, 2012) y el mantenimiento de una activa presencia en redes sociales e Internet (Madrid-Morales, 2017). Este proceso de globalización ha venido impulsado desde las más altas instancias del gobierno y del Partido Comunista Chino (PCCh), que han expresado repetidamente su deseo de que China desempeñe un papel más importante en el mercado mundial de información y entretenimiento. En 2008, Li Changchun, exmiembro del Politburó del Comité Permanente del PCCh a cargo de los medios de comunicación, reflexionó sobre la necesidad de que los medios de comunicación se internacionalizaran en un discurso ante profesionales del sector (C. Li, 2008). Reconoció la falta de influencia de China y pidió esfuerzos para revertir la situación, pidió una política de "salida hacia el exterior" (zou chu qu) de los medios, y señaló la necesidad de profesionalizar los medios para hacerlos más competitivos.

La fuerza que guía este proceso de internacionalización parece ser la percepción generalizada entre la élite China (y parte de la población) de que la opinión pública extranjera no entiende el ascenso de China y que esto se debe, sobre todo, a que los medios extranjeros describen al país en términos negativos (M. Li, 2008). Es en este contexto, el deseo de corregir el aparente desequilibrio entre las representaciones de China en los medios internacionales y la imagen que Pequín quiere transmitir, en donde hay que entender el proceso de "salida hacia el exterior" al que se refería Li Changchun. En la última década, la internacionalización de los medios ha generado un nada desdeñable interés académico, que incluye visiones de conjunto (J. Wang, 2011; X. Zhang, 2013), estudios de caso de diferentes actores (Jirik, 2008; Xin, 2012), análisis del contenido de los medios chinos (S. Li, 2017; Marsh, 2015) y evaluaciones, más o menos fundamentadas, sobre el impacto que están teniendo los esfuerzos del gobierno chino (Huang \& Ding, 2006; Kurlantzick, 2007). Este artículo pretende contribuir a dicho debate, centrando su atención en las acciones de los medios de comunicación chinos en África, la región en donde más activa ha sido la diplomacia pública de Pequín.

\section{África en la política comunicativa de China}

Con motivo del $60^{\circ}$ aniversario del inicio de las relaciones diplomáticas con África en 2006, China organizó en Pequín la 3a Conferencia Ministerial del Foro de Cooperación China-África (FOCAC de sus siglas en inglés), a la que asistieron 41 jefes de estado africanos. El resultado más tangible de la reunión fue el Plan de Acción FOCAC de Pequín, un documento al cual se refieren, todavía hoy con regularidad, la República Popular de China y los gobiernos africanos (Plessis, 2014). El texto incluye una subsección sobre la cooperación chino-africana en el campo de los medios de comunicación. El Plan de Acción pide "que los medios de comunicación jueguen un papel positivo en la mejora de la comprensión mutua y la amistad" y alienta a que haya "más información y cobertura por parte de los medios de comunicación de la otra parte". El texto propone además acciones para facilitar "el envío de corresponsales residentes y no residentes" (FOCAC, 2006) (1). En ningún otro foro multilateral impulsado por China destaca tanto la importancia de los medios de comunicación como en el caso de FOCAC. Para Pequín, el continente africano es un territorio amigo, en donde los esfuerzos de internacionalización de los medios son vistos con menos recelo que en otras partes del mundo. Es por eso que África es el escenario de muchas "primeras veces" para China: CGTN Africa fue el primer centro de producción de televisión fuera de China; Nairobi fue la primera ciudad en donde se abrió una radio FM en chino (China Daily, 2006); swahili fue la lengua escogida para el primer doblaje de una teleserie moderna china (N. Chen, 2013)... 
DANI MADRID-MORALES La internacionalización de los medios de comunicación chinos: un estudio de la producción periodística en CGTN AFRICA.

Tabla 1. Tipología de actividades de empresas y agencias estatales chinas en el sector de los medios y la comunicación en África

\begin{tabular}{|l|l|l|l|}
\hline Tipo de actividad & Industria & Fecha & \multicolumn{1}{|c|}{ Descripción } \\
\hline $\begin{array}{l}\text { Producción de } \\
\text { contenidos }\end{array}$ & Prensa & 2012 & $\begin{array}{l}\text { Publicación de la revista bilingüe } \\
\text { (francés e inglés) Chinafrica por el } \\
\text { Grupo China International Publishing } \\
\text { con sede en Johannesburgo. }\end{array}$ \\
\hline $\begin{array}{l}\text { Distribución de } \\
\text { contenidos }\end{array}$ & Televisión & 2008 & $\begin{array}{l}\text { Establecimiento en Kigali de StarTimes, } \\
\text { una plataforma de televisión digital } \\
\text { (cable, terrestre y satélite) con } \\
\text { presencia en 16 países africanos. }\end{array}$ \\
\hline $\begin{array}{l}\text { Desarrollo de } \\
\text { infraestructuras }\end{array}$ & Móvil & 2013 & $\begin{array}{l}\text { La compañía de telecomunicaciones } \\
\text { china ZTE es seleccionada para la } \\
\text { expansión y la mejora de la red de } \\
\text { telefonía móvil en Etiopía }\end{array}$ \\
\hline Inversión Directa & Prensa & 2013 & $\begin{array}{l}\text { Inversores chinos compran el 20\% } \\
\text { de las acciones del grupo editorial } \\
\text { Independent Media de Suráfrica, el } \\
\text { segundo más importante del país. }\end{array}$ \\
\hline Formación & Múltiples & 2016 & $\begin{array}{l}\text { Periodistas de 13 países africanos } \\
\text { de habla inglesa participan en un } \\
\text { programa de formación durante dos } \\
\text { semanas en universidades de China }\end{array}$ \\
\hline
\end{tabular}

Fuente: Adaptado de Madrid-Morales y Wasserman (2017)

No obstante, sería erróneo dar a entender que la presencia de los medios chinos en África es algo nuevo. Xinhua creó su primera delegación en África subsahariana en la República de Guinea ya en 1959, tras haber operado durante años una sede en Egipto (S. Wang, 2001). Lo que ha cambiado desde entonces ha sido la naturaleza de las relaciones. En los años cincuenta y sesenta, China estaba inmersa en una batalla ideológica en múltiples frente, la mayor parte de la cual tuvo lugar en los medios de comunicación (véase, por ejemplo, Friedman, 2015; Pradet, 1963). Hoy en día, la relación es mucho más diversa, está cada vez más alejada de la retórica ideológica del maoísmo y es, crecientemente, de tipo económico. Como se puede ver en la Tabla 1, en la actualidad, las empresas de comunicación chinas, así como las agencias gubernamentales, están presentes en cinco ámbitos: la creación y distribución de contenidos, el desarrollo de infraestructuras, la inversión directa y la formación. Mientras que algunas de estas actividades se pueden entender como formas de cooperación y ayuda (Gagliardone, Stremlau, \& Nkrumah, 2012), otras están claramente encaminadas a la maximización de intereses económicos y a la creación de narrativas informativas chinas propias. Este es el caso de CGTN Africa.

La emisora, inicialmente conocida como CCTV Africa, empezó a funcionar en enero de 2012 como centro de producción y emisión con sede en Nairobi (Kenia). CGTN Africa produce con- 
tenido para el canal de noticias en inglés de la televisión central china (CGTN), para los informativos en chino $y$, desde hace un tiempo, algunos de sus contenidos se distribuyen también en el canal en lengua francesa. CGTN Africa, que alberga un equipo multinacional, produce y emite cinco programas. Tres de ellos comenzaron a emitirse ya en 2012. Se trata de Africa Live, un informativo diario (con una edición al mediodía y otra por la tarde) que se centra exclusivamente en la actualidad africana; Faces of Africa, una serie documental que ofrece perfiles de individuos africanos de éxito; y Talk Africa, un espacio de entrevistas semanal. En julio de 2014, se añadieron dos nuevos programas a la parrilla: Match Point, que cubre deportes africanos y Global Business, que se centra en noticias económicas y financieras.

La expansión reciente de los medios de comunicación chinos en África ha despertado el interés de parte de la academia. La mayor parte de estudios hasta la fecha se han centrado en analizar el contenido de los medios (por ejemplo, S. Li, 2017; Marsh, 2016; Y. Zhang \& Matingwina, 2016). En un estudio sobre la agencia de noticias Xinhua en África, Xin (2012) concluyó que la agencia trata de diferenciarse de "los medios Occidentales, que se centran principalmente en los "problemas" de los países africanos" (p. 122). Esta idea de que China en África está creando una nueva narrativa que se aleja de la presunta prevalencia de imágenes negativas del continente, ha sido explotada por Pequín para justificar y alabar las actividades de sus medios en el continente. En un discurso en 2014, el entonces ministro de la Administración Estatal General de Prensa, Publicación, Radio, Cine y Televisión de China, Cai Fuchao, dijo de los medios de comunicación chinos en África, incluyendo CGTN, que estos "adoptarán un enfoque realista y pragmático, contando la verdad, proponiendo soluciones pragmáticas, realizando un trabajo práctico y buscando resultados" (Cai, 2014).

En el caso específico de CGTN Africa, aunque existen algunos estudios basados en entrevistas y observación no participante de la redacción (Gagliardone, 2013; X. Zhang, 2013), estos se refieren a los meses inmediatamente posteriores a su creación y no reflejan los cambios que se han ido produciendo a medida que las dimensiones de la redacción han ido creciendo. Además, estos estudios ofrecen una visión general de las operaciones de la cadena, pero apenas consiguen entrar a analizar el proceso de producción periodística. Por lo tanto, a día de hoy no disponemos de un relato académico sistemático de cómo trabajan los periodistas de CGTN Africa ni sobre cuáles son las dinámicas profesionales dentro de la redacción. En este sentido, parece relevante formular las siguientes preguntas para guiar esta investigación y completar los estudios ya existentes:

1 - ¿Cómo se organiza y estructura la redacción de CGTN Africa?

2 - ¿Cuáles son los valores noticia predominantes en la redacción de CGTN Africa?

3 - ¿Qué impacto ha tenido la llegada de CGTN Africa sobre la actividad periodística en el continente?

\section{MÉTODO}

Este artículo se basa en 36 entrevistas(2) en profundidad realizadas en Nairobi (Kenia) y Pequín (China) entre febrero y noviembre de 2015, y media docena de visitas cortas a las oficinas de CGTN Africa(3). En la selección de informantes se usó una combinación de muestreo de conveniencia y "por avalancha". El autor usó contactos previos para acceder a media docena de informantes que, a su vez, sugirieron a otros informantes. También se usaron redes sociales 
(Twitter y LinkedIn) para contactar a algunos extrabajadores. En la selección, se tuvieron en cuenta criterios de representatividad de diferentes categorías profesionales y nacionalidades. Seis personas se negaron a ser entrevistadas y 28 no respondieron a la solicitud. CGTN Africa organizó tres de las entrevistas, mientras que las otras se hicieron fuera de las instalaciones de la cadena.

La muestra final incluye 18 hombres y 18 mujeres, de los cuales 16 son kenianos, 7 chinos, 4 sudafricanos y el resto proviene de otros países africanos, Europa y América del Norte. Entre los entrevistados hay presentadores (3), guionistas (3), periodistas (4), productores (6), editores (2), supervisores (3), corresponsales (10), un becario, un operador de cámara y un alto directivo. Excepto las entrevistas a corresponsales fuera de Nairobi, todas las demás se realizaron cara a cara y duraron entre 26 y 81 minutos, con una duración media de 51 minutos. Todas las entrevistas, con excepción de dos, fueron grabas y transcritas a posteriori para su análisis. Los entrevistados dieron su consentimiento informado para realizar las grabaciones y aceptaron participar en el estudio. A todos ellos se les garantizó anonimato, por lo que cualquier referencia que pudiera llevar a su identificación ha sido eliminada de este texto.

Las entrevistas fueron semiestructuradas. A todos los informantes se les hicieron preguntas similares, aunque el orden en que se les plantearon las cuestiones difirió de una entrevista a otra. El cuestionario se organizó en torno a siete bloques y, siguiendo a Kvale (2007), varias preguntas clave fueron identificadas en cada uno de los bloque: (1) historia personal (por ejemplo, ¿por qué decidió unirse a CCTV Africa?); (2) naturaleza de las tareas asignadas (p. ej., pensando en su última asignación, ¿cómo preparó la crónica?); (3) valores noticia y normas periodísticas (p. ej., ¿puede explicarme la política editorial de CCTV?); (4) relaciones interpersonales en la redacción (p. ej., ¿cómo describiría su relación con sus colegas?); (5) relaciones profesionales fuera de la redacción (p. ej., ¿interactúa usted con otros periodistas que trabajan en otros medios chinos?); (6) opiniones sobre el sistema de medios chino (p. ej., ¿hasta qué punto conocía usted los medios chinos antes de unirse a CCTV Africa?); y, (7) opiniones sobre la presencia de China en África (p. ej., ¿por qué cree que CCTV está invirtiendo en los medios de comunicación de África?).

Para analizar los datos, las entrevistas fueron codificadas temáticamente utilizando QSR NVivo, un software de análisis de datos cualitativo que ayuda a dar sentido y a teorizar a partir de grandes cantidades de información textual (Bazeley \& Jackson, 2013). De los múltiples temas identificados, este artículo se centra en dos: la estructura de la redacción y los valores noticia de CGTN Africa.

\section{RESULTADOS}

El presente artículo da respuesta a tres preguntas, dos centradas en el proceso de producción periodística en CGTN Africa, y una tercera referida al impacto de la internacionalización de los medios chinos. Se ha optado por dividir el apartado de "Resultados" en dos partes. En la primera, se describe la estructura y organización de la redacción, y en la segunda, se habla de los valores noticia. Se da respuesta a la tercera pregunta en el apartado "Discusión". 
DANI MADRID-MORALES La internacionalización de los medios de comunicación chinos: un estudio de la producción periodística en CGTN AFRICA.

\subsection{Organización y estructura de la redacción}

CGTN Africa es el nombre comercial del centro de transmisión y producción de CCTV en Nairobi (Kenia). Un centro similar, llamado CGTN America, se inauguró en Washington (Estados Unidos) a principios de 2012 y, según algunos entrevistados (Entrevista 39XX-ZLQ), existen planes para establecer un tercero en Londres (CGTN Europe)(4). Los centros de Nairobi y Washington están afiliados al canal madre, CGTN, que es el canal de noticias de 24 horas en inglés de la televisión central de China y son responsables de producir y difundir dos horas de contenido todos los días de la semana. Las oficinas de CGTN Africa son también la base de operaciones de un equipo de corresponsales chinos, que trabajan bajo la supervisión del editor jefe. Su trabajo, no obstante, no es informar para los canales internacionales de CGTN, sino para las cadenas de televisión domésticas en chino.

Desde finales de 2011, CGTN Africa ocupa parcialmente dos edificios en el distrito de Kilimani de Nairobi. La redacción principal y las oficinas de los directivos de la cadena, y los estudios, la sala de control y los equipos de edición están ubicados en dos pisos separados en un mismo edificio. El segundo edificio incluye una sala de reuniones y una redacción más pequeña, donde trabajan los equipos de los programas semanales. Es en este segundo edificio donde tienen su espacio de trabajo los corresponsales chinos, que raramente interactúan con los redactores del canal en inglés. Los dos edificios no están conectados, pero es posible desplazarse de uno a otro a pie. La desconexión (no solo física, sino también profesional) entre el equipo chino y el internacional quedó clara a partir de mi observación de la actividad periodística en las redacciones y fue confirmada por diversos informantes.

Aunque la primera emisión de CGTN Africa tuvo lugar el 12 de enero de 2012, los preparativos se iniciaron durante el verano de 2011, cuando un grupo de periodistas y editores chinos inició el proceso de selección de periodistas locales (Entrevista 63XX-HLP). El primer equipo de CGTN Africa incluía una combinación de periodistas kenianos experimentados y bien conocidos, como Beatrice Marshall, Robert Soi y Celestine Karoney, y algunos recién graduados. Durante los meses previos al lanzamiento, los periodistas locales recibieron una formación rigurosa por parte del personal chino. Los cursos incluían una introducción básica al periodismo en televisión (redacción de guiones, edición de vídeo...), así como formación sobre equipos de video, historia y cultura chinas. La idea de que CGTN Africa debía ofrecer una visión positiva sobre África se transmitió repetidamente durante la etapa formativa. Según uno de los primeros reporteros en unirse, "lo que nos contaron fue que, cuando decidieron crear CCTV Africa, dijeron: 'queremos historias positivas de África'"' (Entrevista 56XX-LQN). La formación para las personas contratadas tras el lanzamiento de la cadena fue mucho menos consistente y varios informantes aseguraron no haber recibido instrucciones específicas sobre lo que se esperaba de ellos.

La jerarquía parece ser importante en la redacción, aunque tanto los directivos de la cadena, así como los supervisores chinos en la redacción se mostraron bastante reacios a hablar sobre esta cuestión. Varios periodistas y editores, sin embargo, se refirieron a la complejidad de la jerarquía y a la dificultad de navegar a través de los diferentes niveles de gestión y supervisión. Un guionista comparó la redacción con el reino animal: los gerentes y administradores serían leones, y los redactores, simples cebras (Entrevista 16XX-LQG). 
DANI MADRID-MORALES La internacionalización de los medios de comunicación chinos: un estudio de la producción periodística en CGTN AFRICA.

La redacción está verticalmente estructurada en la parte superior y tiene una organización horizontal amplia en la base. Los niveles superiores de la jerarquía los ocupan ciudadanos chinos. Todos tiene experiencia previa trabajando en la sede de CCTV en Pequín y son enviados, voluntariamente, a Kenia por un período limitado de tiempo, alrededor de tres años. Con excepción de los tres puestos más altos de la jerarquía (director de la delegación, el editor jefe y el editor adjunto), el resto del equipo directivo es muy joven y CGTN Africa es su primer destino laboral en el extranjero. Un sistema de rotación está en vigor en CCTV, con los diferentes destinos en el extranjero clasificados en tres niveles (A, B, C); los países africanos están en el nivel $C$. Haber sido destinado a un país africano hace que sea más fácil luego poder pedir el traslado a un país en el nivel $\mathrm{A}$.

El número de trabajadores chinos en la redacción oscila entre 20 y 35, ya que el tamaño del equipo de corresponsales estacionados en Nairobi cambia muy a menudo (Entrevista 25XXHFX). El resto del personal, entre 90 y 100 personas, incluye presentadores, productores, guionistas, editores, planificadores, reporteros, editores de video, ingenieros, directores, camarógrafos y otros puestos técnicos, además de un equipo administrativo. Todo el personal no chino es contratado localmente por una compañía llamada Bright Vision Media Ltd., una subsidiaria de la Corporación de Televisión Internacional de China (CITVC), con sede en Kenia. La cadena también da trabajo a más de veinte corresponsales extranjeros independientes, algunos de los cuales trabajan exclusivamente para CGTN y algunos que trabajan para otros medios de comunicación, en una docena de países africanos.

Esta estructura jerárquica se traduce en múltiples capas de supervisión durante la producción periodística. Las dos ediciones diarias del informativo Africa Live son coordinadas por un editor, encargado de redactar los titulares del boletín, asignar historias a redactores y reporteros, y coordinar con el equipo de asignaciones, que mantiene contacto con los corresponsales. Desde 2012, CGTN Africa ha contratado a varios editores experimentes de Kenia, Sudáfrica y Estados Unidos. El jefe de redacción, que en el momento de realizar el trabajo de campo era un británico con una extensa carrera profesional en medios de comunicación sudafricanos, supervisa las operaciones diarias en la redacción bajo la mirada más o menos atenta de un supervisor chino. Según los editores, hay muy poca interferencia por parte del personal chino durante el proceso de preparación del informativo. Sin embargo, estos supervisores sí que participan regularmente en las reuniones semanales y diarias de planificación, donde se proponen temas y se toman decisiones sobre la asignación de recursos. Uno de los supervisores chinos tiene una teleconferencia diaria con la redacción de Pequín, durante la cual recibe instrucciones sobre asuntos que necesitan un trato preferencial.

Como es común en la mayoría de redacciones, antes de locutar una noticia, los guiones, incluidos los que mandan los corresponsales, son revisados por un editor. En ciertas ocasiones, como cuando se trata de noticas sobre China, los guiones son corregidos por el editor adjunto o el propio editor jefe. Antes de cada boletín, todo el contenido del programa pasa por una última revisión a cargo de algún miembro del personal chino. Ninguno de los informantes fue capaz de recordar una sola historia que hubiera sido vetada en el último minuto. Sin embargo, la autocensura parece ser común. Varios informantes afirmaron saber cuáles eran los límites de lo que era aceptable y qué líneas no debían cruzar. Un ex corresponsal lo resumió en estos términos: 
DANI MADRID-MORALES La internacionalización de los medios de comunicación chinos: un estudio de la producción periodística en CGTN AFRICA.

Yo me auto-censuraba. Hubo un par de veces cuando querían cambiar mi guion por razones que creo que eran bastante innecesarias, pero lo hicieron. En términos de censura, nunca tuve mucho problema. Creo que sabían que yo sabía lo que querían. Así que en realidad no necesitaban censurarme (Entrevista 21XX-VKA).

\subsection{Los valores noticia en CGTN Africa}

Aunque periódicamente la redacción recibe desde la sede de CCTV en Pequín instrucciones sobre qué temas cubrir, la mayoría de historias son propuestas por los corresponsales, los productores y los propios redactores. Al igual que en otras cadenas de televisión, la disponibilidad de material en video sobre aquello que se quiere explicar determina qué noticias se tratan y cuáles se aparcan. CGTN Africa recibe imágenes de las agencias internacionales AP Television News, Reuters y HLA. No existe, sin embargo, un acuerdo con el servicio de video de la agencia de noticias Xinhua, ni tampoco con la cadena de televisión CNC, también operada por Xinhua, y que tienen una amplia presencia en África. Pese a que ambos son medios estatales y comparten un mandato político similar, la cooperación entre las dos empresas es escasa. CGTN Africa tampoco recibe los teletipos de Xinhua, a pesar de que los redactores los usan (a través de la página web de la agencia) cuando escriben noticias sobre China.

Los profesionales de la información en CGTN Africa, como la mayoría de sus colegas en los medios de comunicación de otros países, se encuentran con dificultades a la hora de ponerse de acuerdo sobre qué convierte un evento en algo noticioso. Durante las entrevistas, en varias ocasiones los periodistas recurrieron al cliché "una noticia es una noticia". Uno de los presentadores de la cadena aseguró con firmeza que en CGTN África: "sabemos exactamente dónde queremos estar, cómo queremos que nos vean, cómo queremos sonar y lo que queremos que el mundo piense de nosotros" (Entrevista 39XX-ZLQ). La incapacidad de los periodistas para hacer un listado de valores noticia no significa que la selección de las noticias se haga al azar, pero es revelador que, como otros han señalado en el pasado (Gans, 1979), un grado sustancial de subjetividad entra en el proceso. En CGTN Africa, la selección de noticias está en gran medida en manos del jefe de redacción (británico), los planificadores (kenianos) y los editores (kenianos y sudafricanos), cuyo trabajo está bajo la supervisión más o menos regular del editor adjunto y el supervisor de la redacción (ambos chinos).

Sólo dos valores noticia parecen ser compartidos por toda la redacción: la "proximidad geográfica" y la "positividad". Hay un tercer valor-noticia, "conflicto", que es citado también de forma bastante regular. El área geográfica de interés de CGTN Africa es, como cabría esperar, el continente africano y por lo tanto el valor-noticia "proximidad" se refiere a los acontecimientos que tienen lugar en África o aquellos en los que personas u organizaciones africanas están involucradas. El valor-noticia "positividad" toma múltiples formas, pero generalmente parece referirse a historias de éxito (individuos que logran sus metas, empresas que consiguen mejorar sus resultados económicos...), soluciones a problemas duraderos (curas médicas, desarrollo de infraestructura, fin de conflictos bélicos...) y, más en general, noticias sobre la vida cotidiana. Uno de los entrevistados utilizó el siguiente ejemplo para explicar lo que significa "positividad" como valor-noticia en CGTN África:

Estuve en Abuja [en Nigeria] para las elecciones. [La organización terrorista] Boko Haram no está en Abuja. Abuja es una ciudad bulliciosa. Estaban muy preocupados por la seguridad porque era hora de las elecciones. Nosotros quisimos mostrar las medidas 
de seguridad que el gobierno había puesto en marcha para permitir que la gente votase, en contraposición con los desafíos de seguridad que podrían hacer que la gente no fuese a votar. La diferencia entre una opción y la otra es que, nosotros dijimos: 'El gobierno ha hecho esto para que la gente salga a votar. Es una democracia; deben salir y elegir a su líder. (Entrevista 39XX-ZLQ).

Desde un primer momento, la dirección de CGTN Africa animó a los periodistas a contar una historia positiva del continente, lo que explicaría la prevalencia del valor-noticia "positividad". No obstante, la agenda informativa del canal se ha ido ampliando a medida que los periodistas y editores locales han ido tratando de ensanchar las fronteras informativas. Un reportero lo explicaba de esta forma:

esto de las noticias positivas era algo muy interesante de intentar hacer, porque cualquiera que esté en el mundo del periodismo, cualquiera que trabaje en periodismo dirá que: 'las noticias son noticias.' No se puede decir que no informaré sobre la crisis en Sudán del Sur porque no es positivo. Tardó tres meses, tres meses después de que comenzáramos las emisiones, ya estábamos siguiendo la norma de que las noticias son noticias (Entrevista 56XX-LQN).

El hecho de que los altos cargos de CGTN Africa no se involucren activamente en la selección de noticias ha dado espacio para que los editores y redactores adopten una línea editorial propia o moldeen los límites de lo informable. Un productor, por ejemplo, lo veía de esta forma: "no nos engañemos, hay muchos problemas en el continente africano, con lo que, para mí, estas cuestiones tienen una alta prioridad en el boletín informativo" (Entrevista 05XX-OBV).

En resumen, la "positividad" y el "conflicto" coexisten en la redacción como consecuencia de una evolución natural de los objetivos de la cadena. Las ideas y los objetivos del canal, los que fueron encargados a los empleados chinos, han ido siendo moldeados a la vez que el personal local e internacional ha ido promoviendo su versión de cómo debía ser el canal. A finales de 2015, algo más de tres años después del lanzamiento del canal, cuando pregunté a un miembro del equipo de gestión sobre qué tipo de noticias CGTN África estaba cubriendo, simplemente dijo: " si es algo importante e interesante, definitivamente lo incluiremos. Si es interesante y controvertido al mismo tiempo, sí, también lo incluiremos" (Entrevista 63XX-HLP).

Para muchos en CGTN Africa, la voluntad de dar prioridad a aquellas noticias positivas en detrimento de las historias que hablen de conflictos, hambre y desastres naturales, es vista como una reacción lógica a años de cobertura negativa del continente en los medios de comunicación de todo el mundo. Así lo explicaba un veterano de la redacción:

Estamos haciendo esto para corregir un error. Porque, seguramente, después de cincuenta años de independencia en África, el resto del mundo sigue pensando que todavía estamos, no sé, arrastrándonos y luchando. Y, por supuesto que no es así: ¡¡hemos avanzado! (Entrevista 39XX-ZLQ)

En más de una docena de entrevistas, los informantes compararon la cobertura informativa de CGTN Africa y la del resto de medios de comunicación chinos con la de los medios "occidentales" para mostrar que, a diferencia de la cobertura predominantemente negativa de África que se supone que se ha hecho desde estas organizaciones mediáticas, los medios chinos "siem- 
DANI MADRID-MORALES La internacionalización de los medios de comunicación chinos: un estudio de la producción periodística en CGTN AFRICA.

pre encuentran algún lado bueno de África para mostrar al público" (Entrevistas 00XX-VYY).

\section{DISCUSIÓN}

Del análisis de las entrevistas, se desprenden al menos dos ideas sobre las que reflexionar. La primera tiene que ver con el modelo de periodismo que se practica en la redacción de CGTN Africa, un medio de titularidad china, pero con muchas particularidades si se compara con los medios de comunicación de ámbito doméstico. Tanto Gagliardone y Nyíri (2016) como Zhang (2013) habían mostrado previamente que el periodismo en CGTN Africa y el de otros medios extranjeros escapa las clasificaciones existentes sobre periodismo en China (Hassid, 2011; Pan \& Lu, 2009). En la redacción de CGTN Africa se practica un híbrido periodístico que combina elementos de diferentes tradiciones periodísticas en China con una concepción africana y global del periodismo, que aportan los profesionales contratados localmente. Se trata de un periodismo pragmático. Sin dejar de ser conscientes que su trabajo es el de portavoces de un gobierno, el chino, los periodistas aprovechan los recursos a su disposición para avanzar una agenda propia ya que gozan de espacio para maniobrar entre las normas y la estructura imperantes.

La segunda idea, es que las dimensiones nacional e internacional de los medios de comunicación chinos difieren en mucho más de lo que a menudo reflejan los trabajos académicos. A pesar de que todos los medios de comunicación chinos con presencia internacional son de propiedad estatal y están bajo férreo control, existen una serie de dinámicas-como se ha visto en este artículo-que crean un marco de actuación periodística distinto. Esto se extiende a los valores noticia. La idea que CGTN Africa tiene que contar noticias positivas sobre el continente aparece de forma recurrente en las entrevistas. De hecho, es una idea que ya habían apuntado otros autores previamente (Gagliardone, 2013; Wasserman, 2013). No obstante, hay un cierto distanciamiento entre la manera como los periodistas de CGTN Africa reflexionan acerca del concepto de "positividad" y los intentos que se han hecho con anterioridad de conectar el periodismo de los medios de comunicación chinos en África con ciertos modelos alternativos de periodismo, como serín el periodismo de paz (peace journalism), el periodismo al servicio del desarrollo (developmental journalism) o el periodismo constructivo (constructive journalism). En contra de lo que sostienen Zhang y Matingwina (2016), no hay ninguna mención a ninguno de estos paradigmas periodísticos en las entrevistas. Sí que existe, en cambio, un discurso de oposición binaria entre "nosotros" (los periodistas africanos y chinos) y "ellos" (los periodistas de los medios europeos y norteamericanos), que sirve para justificar el uso del valor-noticia "positividad": África ha sido contada de forma demasiado negativa por "ellos" y, en consecuencia, ahora es necesario que "nosotros" corrijamos la narrativa.

Aunque los estudios que se han hecho hasta el momento sobre el contenido de los medios de comunicación chinos en África (S. Li, 2017; Marsh, 2015, 2016; X. Zhang, 2013) parecen señalar que, en realidad, el valor-noticia "positividad" es mucho menos importante de lo que cabría esperar, CGTN Africa, y también Xinhua y otros medios similares, han conseguido presentarse como abanderados de una nueva forma de contar la realidad africana que entronca con la llamada narrativa de "África en ascenso" (Nothias, 2014). La de CGTN Africa, en contra de lo que pudiera pensarse, no es una narrativa controlada férreamente por el gobierno, sino el producto de una negociación constante entre una estructura jerárquica y rígida propia de un medio de titularidad pública en un régimen autocrático de ideología comunista, y la agencia de periodis- 
tas con inquietudes y agendas propias, que alejados de la supervisión de Pequín y dentro de una redacción en donde la supervisión existe, pero se ejerce de forma desigual, pueden informar una agenda que satisface al mismo tiempo los deseos de explicar África al mundo desde una perspectiva africana que tienen los periodistas locales, y los deseos de Pequín de presentarse como una voz alternativa y positiva en los flujos informativos globales sobre África. A este producto se le podría poner la etiqueta de "noticias sobre África con características chinas".

La existencia de controles en CGTN Africa es innegable, particularmente en las historias sobre China o las que se consideran sensibles a los intereses de la política exterior, pero como dice el proverbio chino: "Las montañas son altas y el emperador está muy lejos". Mientras que los líderes chinos esperan que CGTN Africa, así como otros medios que operan en el extranjero, ayuden a propagar la política exterior china, lo que está sucediendo en realidad es que China está dando la oportunidad (quizás de forma involuntaria) a periodistas africanos para que cuenten su propia historia y la historia que quieren contar es la de un continente africano en ascenso, que tiene problemas y muchas cosas por mejorar, pero que crece y mejora. Esta narrativa, la de las "noticias sobre África con características chinas", es en realidad pocas veces una narrativa china; es más bien una narrativa africana, expresada a través de un medio de comunicación chino, y que ha sido construida en oposición a la percepción de que existe una narrativa predominante en los medios globales de un continente africano en ruinas.

\section{CONCLUSIONES}

El proceso de internacionalización de los medios de comunicación chinos que se inicia a mediados de los años noventa, ha sido especialmente intenso en el continente africano. Este artículo complementa los estudios existentes sobre este proceso y, en particular sobre la cadena de televisión CGTN Africa, con un estudio sobre la producción periodística en una redacción multinacional china que incluye a profesionales no sólo chinos, sino también africanos, europeos y norteamericanos. El artículo ha demostrado la complejidad de dar sentido a las prácticas periodísticas en un lugar de trabajo culturalmente heterogéneo, dando voz a los periodistas.

El análisis de las entrevistas ha revelado que la idea de "positividad" como valor-noticia está bastante generalizada en la redacción. A pesar de que su importancia no es compartida por todos, se puede afirmar que, en CGTN Africa, las noticias positivas son también noticia, en contra de la aparente lógica imperante en el ámbito periodístico de que sólo aquello "negativo" es noticiable. El artículo, además, aporta detalles sobre el modelo organizativo. En la descripción de la estructura de la redacción se ha destacado el contraste entre la organización vertical de la gestión (ocupada por trabajadores chinos) y la estructura horizontal amplia de las posiciones no directivas (todas ellas ocupadas por extranjeros). Si bien la redacción es altamente jerárquica, se demuestra que hay un cierto nivel de descentralización en las operaciones diarias, lo que permite un grado considerable de flexibilidad y empodera la agencia de los redactores y editores.

Las redacciones son instituciones sociales complejas y este artículo sólo ha cubierto parcialmente una faceta del proceso de producción periodística. Faltaría tener en cuenta en estudios futuros otras cuestiones como las dinámicas de poder, el género y la diversidad racial. Todos estos temas aparecieron de forma regular en las entrevistas. Quizás el principal elemento a 
mejorar de este estudio es la dependencia de entrevistas como fuente de información. Sin embargo, como se señaló anteriormente, el acceso a la redacción de CGTN Africa no es fácil. Los medios de comunicación chinos son, en general, pero particularmente la radio y la televisión, organizaciones opacas y reacias a abrirse al escrutinio; más aún al escrutinio de un académico joven, blanco y europeo.

Con todas sus carencias, este trabajo ofrece una triple contribución al estudio del periodismo, y abre varias avenidas para estudios futuros. En el estudio de los valores-noticia, se ha mostrado la adaptabilidad de los criterios de selección de noticias. En el estudio del periodismo en China, se ofrece una primera visión de cómo se practica el periodismo fuera de las redacciones domésticas. Y, por último, en las discusiones sobre comunicación internacional, se ha puesto el foco en los intercambios comunicativos entre actores del Sur Global, un área de investigación que sigue siendo algo olvidada en los estudios de comunicación.

Para una organización con apenas cinco años de antigüedad, CGTN Africa se ha visto expuesta a un grado inusualmente alto de escrutinio. Este artículo es, sin embargo, uno de los primeros en dar extensa voz a los periodistas de la cadena. De hecho, si algo falta en el estudio de la internacionalización de los medios de comunicación chinos son más trabajos etnográficos. Hay un déficit claro de estudios que se centren en los mensajeros y cómo estos dan sentido a su trabajo en la redacción. Del mismo modo, parece urgente conectar el estudio de los mensajes, que es predominante en la investigación sobre CGTN Africa, a los procesos de recepción por parte de la audiencia. Este artículo ofrece elementos sobre los que poder desarrollar investigaciones futuras en estas dos áreas.

\section{NOTAS}

(1) El Plan de Acción de la 4a Conferencia Ministerial de Sharm El Sheikh en 2009 y el de la

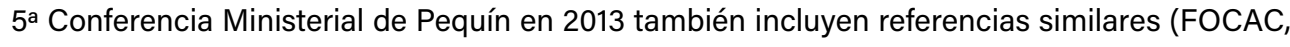
2009, 2013).

(2) El tamaño de la muestra es similar al usado en otros estudios sobre producción periodística basados enteramente en entrevistas (Archetti, 2013, 25 entrevistas; Bunce, 2010, 23 entrevistas; Harries \& Wahl-Jorgensen, 2007, 20 entrevistas).

(3) Estas visitas nunca duraron más de dos horas y el personal de CGTN Africa siempre me acompañó. Presenté varias solicitudes para realizar observación no participante en la redacción, pero todas fueron rechazadas por la dirección.

(4) Además de los dos centros de producción en Washington y Nairobi, CGTN también tiene sedes regionales en Dubái, Hong Kong, Moscú y Río de Janeiro. Todas estas, sin embargo, son de tamaño menor.

\section{REFERENCIAS BIBLIOGRÁFICAS}

- $\quad$ AP. (2016, diciembre 31). CCTV gets makeover in push overseas. South China Morning Post. Recuperado a partir de http://www.scmp.com/news/china/policies-politics/article/2058429/chinas-state-broadcaster-cctv-rebrands-international

- Archetti, C. (2013). Journalism in the age of global media: The evolving practices of foreign correspondents in London. Journalism, 14(3), 419-436. https://doi. org/10.1177/1464884912472140

- Barboza, D. (2009, enero 14). News Media Run by China Look Abroad for Growth. The 
DANI MADRID-MORALES La internacionalización de los medios de comunicación chinos: un estudio de la producción periodística en CGTN AFRICA.

New York Times. Recuperado a partir de http://www.nytimes.com/2009/01/15/business/ worldbusiness/15tele.html

- $\quad$ Bazeley, P., \& Jackson, K. (2013). Qualitative data analysis with NVivo. Thousand Oaks: SAGE.

- Bunce, M. (2010). «This Place Used to be a White British Boys' Club»: Reporting Dynamics and Cultural Clash at an International News Bureau in Nairobi. The Round Table, 99(410), 515-528. https://doi.org/10.1080/00358533.2010.509950

- $\quad$ Cai, F. (2014, junio). Speech to the Second Forum on China-Africa Media Cooperation. Presentado en Second Forum on China-Africa Media Cooperation, Beijing. Recuperado a partir de http://english.cntv.cn/2014/06/16/ARTI1402882926579516.shtml

- CGTN Africa. (2017). CGTN Africa (CCTV Africa) celebrates 5th anniversary in colorful ceremony. Recuperado a partir de https://www.youtube.com/watch?v=QLKjBRjamiA

- $\quad$ Chang, T.-K., \& Lin, F. (2014). From propaganda to public diplomacy: Assessing China's international practice and its image, 1950-2009. Public Relations Review, 40(3), 450-458. https://doi.org/10.1016/j.pubrev.2014.04.008

- $\quad$ Chang, W. H. (1989). Mass media in China: the history and the future. Ames: lowa State University Press.

- $\quad$ Chen, A., \& Wu, V. (2009, enero 12). Beijing in 45b yuan global media drive. South China Morning Post. Recuperado a partir de http://www.scmp.com/article/666847/beijing-45byuan-global-media-drive

- Chen, C. C., Colapinto, C., \& Luo, Q. (2010). China Radio International in the Digital Age: Propagating China on the Global Scenario. Global Media Journal, 9(16).

- Chen, N. (2013, mayo 25). Chinese TV series find a new voice in Africa. China Daily. Recuperado a partir de http://usa.chinadaily.com.cn/china/2013-05/25/content_16530478.htm

- China Daily. (2006, diciembre 4). For 65 years, a window to the world. China Daily, p. 1.

- Culbert, D. (2010). Public Diplomacy and The International History of Mass Media: The USIA, The Kennedy Assassination, and The World. Historical Journal of Film, Radio and Television, 30(3), 421-432. https://doi.org/10.1080/01439685.2010.505041

- Cull, N. J. (2008). Public Diplomacy: Taxonomies and Histories. The ANNALS of the American Academy of Political and Social Science, 616(1), 31-54. https://doi. org/10.1177/0002716207311952

- FOCAC. (2009). Forum on China-Africa Cooperation Sharm El Sheikh Action Plan (20102012). Recuperado 14 de octubre de 2013, a partir de http://www.fmprc.gov.cn/zflt/eng/ zxxx/t280369.htm

- FOCAC. (2013). Forum on China-Africa Cooperation Beijing Action Plan (2013-2015). Recuperado 14 de octubre de 2013, a partir de http://www.fmprc.gov.cn/zflt/eng/zxxx/ t280369.htm

- $\quad$ Friedman, J. S. (2015). Shadow Cold War: the Sino-Soviet competition for the Third World. Chapel Hill: University of North Carolina Press.

- Gagliardone, I. (2013). China as a persuader: CCTV Africa's first steps in the African mediasphere. Ecquid Novi: African Journalism Studies, 34(3), 25-40. https://doi.org/10.1080/02 560054.2013.834835

- $\quad$ Gagliardone, I., \& Nyíri, P. (2016). Freer but not free enough? Chinese journalists finding their feet in Africa. Journalism. https://doi.org/10.1177/1464884916636171

- $\quad$ Gagliardone, I., Stremlau, N., \& Nkrumah, D. (2012). Partner, prototype or persuader? China's renewed media engagement with Ghana. Communication, Politics \& Culture, 45, 174-196.

- Gans, H. J. (1979). Deciding what's news: a study of CBS evening news, NBC nightly news, 
DANI MADRID-MORALES La internacionalización de los medios de comunicación chinos: un estudio de la producción periodística en CGTN AFRICA.

Newsweek, and Time. Ann Arbor: University of Michigan Press.

- Gilboa, E. (2008). Searching for a Theory of Public Diplomacy. The ANNALS of the American Academy of Political and Social Science, 616(1), 55-77. https://doi. org/10.1177/0002716207312142

- $\quad$ Gorfinkel, L., Joffe, S., Van Staden, C., \& Wu, Y.-S. (2014). CCTV's Global Outreach: Examining the Audiences of China's «New Voice» on Africa. Media International Australia, 151(1), 81-88. https://doi.org/doi: 10.1177/1329878X1415100111

- Harries, G., \& Wahl-Jorgensen, K. (2007). The culture of arts journalists: Elitists, saviors or manic depressives? Journalism, 8(6), 619-639. https://doi.org/10.1177/1464884907083115

- Hassid, J. (2011). Four Models of the Fourth Estate: A Typology of Contemporary Chinese Journalists. The China Quarterly, 208, 813-832. https://doi.org/10.1017/S0305741011001019

- Huang, Y., \& Ding, S. (2006). Dragon's underbelly: An analysis of China's soft power. East Asia, 23(4), 22-44. Recuperado a partir de http://dx.doi.org/10.1007/BF03179658

- Jacobs, A. (2012, agosto 17). Live From Nairobi, China Puts Its Stamp on News in Africa. The New York Times, p. A1.

- Jirik, J. (2008, mayo). Making news in the People's Republic of China: The case of CCTV-9. (Tesis doctoral) The University of Texas, Austin.

- Jirik, J. (2010). 24-Hour Television News in the People's Republic of China. En S. Cushion \& J. Lewis (Eds.), The Rise of 24-Hour News Television: Global Perspectives (pp. 281-298). Nueva York: Peter Lang.

- Kurlantzick, J. (2007). Charm Offensive: How China's Soft Power Is Transforming the World. New Haven: Yale University Press.

- $\quad$ Kvale, S. (2007). Doing interviews. Thousand Oaks: SAGE.

- Lefkowitz, M. (2017). Chinese Media, Kenyan Lives: An Ethnographic Inquiry into CCTV Africa's Head Offices (Working Paper No. 2017/9). Washintgon: China-Africa Research Initiative, School of Advanced International Studies, Johns Hopkins University. Recuperado a partir de http://www.sais-cari.org/publications

- $\quad$ Li, C. (2008, diciembre 23). Zai jinian Zhongguo dianshi shiye dansheng ji zhongyang dianshitai jiantai 50 zhounian dahuishang de jianghua [Discurso en conmemoración de los 50 años del nacimiento de la industria de la televisión en China y de la creación de CCTV]. Guangming Ribao, p. 2.

- Li, M. (2008). China Debates Soft Power. The Chinese Journal of International Politics, 2(2), 287-308. https://doi.org/10.1093/cjip/pon011

- Li, S. (2017). Covering Ebola: a comparative analysis of CCTV Africa's Talk Africa and Al Jazeera English's Inside Story. Journal of African Cultural Studies, 29(1), 114-130. https://doi. org/10.1080/13696815.2016.1188687

- Madrid-Morales, D. (2015). China's International Broadcasting and the Spanish Speaking World. En Q. Luo (Ed.), Global Media Worlds and China (pp. 187-203). Beijing: Communication University of China Press.

- Madrid-Morales, D. (2017). China's Digital Public Diplomacy towards Africa: Actors, Messages and Audiences. En K. Batchelor \& X. Zhang (Eds.), China-Africa relations: building images through cultural co-operation, media representation and on the ground activities. Nueva York: Routledge.

- $\quad$ Madrid-Morales, D., \& Wasserman, H. (2017). Chinese media engagement in South Africa: what is its impact on local journalism? Journalism Studies. https://doi.org/10.1080/146167 0X.2016.1266280 
DANI MADRID-MORALES La internacionalización de los medios de comunicación chinos: un estudio de la producción periodística en CGTN AFRICA.

- Marsh, V. (2015). Mixed messages, partial pictures? Discourses under construction in CCTV's Africa Live compared with the BBC. Chinese Journal of Communication, 1-15. https://doi.org/10.1080/17544750.2015.1105269

- Marsh, V. (2016). Politics in the representation of Africa. Africa through Chinese eyes: new frames or the same old lens? African news in English from China Central Television, compared with the BBC. En M. Bunce, S. Franks, \& C. Paterson (Eds.), Africa's media image in the 21st century: from the «heart of darkness» to «Africa rising» (pp. 177-189). Londres: Routledge.

- Mlilo, N. (2015, noviembre 5). China enhances cooperation with African media industry. Recuperado a partir de http://news.xinhuanet.com/english/2015-11/05/c_134784288.htm

- Nothias, T. (2014). 'Rising', 'hopeful', 'new': visualizing Africa in the age of globalization. Visual Communication, 13(3), 323-339. https://doi.org/10.1177/1470357214530063

- Ollé, M. (2017). Transformaciones del paisaje de la información y la comunicación en China. En D. Madrid-Morales (Ed.), Comunicación y poder en Asia oriental (pp. 67-114). BarceIona: Editorial UOC.

- Pan, Z., \& Lu, Y. (2009). Localizing professionalism: discusive practices in China's media reforms. En C.-C. Lee (Ed.), Chinese Media, Global Contexts (pp. 196-214). Londres: Routledge.

- Passin, H. (1962). China's cultural diplomacy. Nueva York: Frederick A. Praeger.

- Paterson, C. (2013). Information flows in sub-Saharan Africa. Sur le journalisme, About journalism, Sobre jornalismo, 2(1), 80-87.

- Plessis, A. D. (2014). The Forum on China- Africa Cooperation, Ideas and Aid: National Interest(s) or Strategic Partnership? Insight on Africa, 6(2), 113-130. https://doi. org $/ 10.1177 / 0975087814535440$

- Pradet, G. (1963). La Russie sovietique et la Chine en Afrique: beaucoup de propaganda, peu d'aide. París: Creátions de presse.

- Shambaugh, D. L. (2013). China goes global: the partial power. Oxford: Oxford University Press.

- Snow, N. (2009). Rethinking Public Diplomacy. En N. Snow \& P. M. Taylor (Eds.), Routledge handbook of public diplomacy (pp. 3-11). Nueva York: Routledge.

- Üngör, Ç. (2009). Reaching the distant comrade Chinese Communist propaganda abroad (1949--1976) (Tesis doctoral). State University of New York, Binghamton.

- USIA. (1973). The external information and cultural relations programs of the People's Republic of China. Washington: United States Information Agency, Research Office.

- Wang, J. (Ed.). (2011). Soft Power in China: Public Diplomacy through Communication. Nueva York: Palgrave Macmillan.

- Wang, S. (2001). Diyipi hei feizhou fenshe de jianli [El establecimiento de la primera oficina en África subsahariana]. En D. He (Ed.), Lishi de Zuji - Xinhuashe 70 Zhounian Huiyi Wenxuan (1931-2001) [Recuerdos de la Historia-Colección de Memorias de los 70 Años de Xinhua (1931-2001)] (pp. 620-627). Pequín: Xinhua Press.

- Wasserman, H. (2013). China in Africa: The implications for journalism. Ecquid Novi: African Journalism Studies, 34(3), 1-5. https://doi.org/10.1080/02560054.2013.861955

- Wekesa, B. (2017). New directions in the study of Africa-China media and communications engagements. Journal of African Cultural Studies, 29(1), 11-24. https://doi.org/10.1080/136 96815.2016.1270197Xin, X. (2012). How the market is changing China's news: the case of Xinhua News Agency. Lanham: Lexington Books. 
- Zhang, X. (2013). How ready is China for a China-style world order? China's state media discourse under construction. Ecquid Novi: African Journalism Studies, 34(3), 79-101. https://doi.org/10.1080/02560054.2013.834834

- Zhang, Y., \& Matingwina, S. (2016). Constructive Journalism: A New Journalistic Paradigm of Chinese Media in Africa. En X. Zhang, H. Wasserman, \& W. Mano (Eds.), China's Media and Soft Power in Africa: Promotion and Perceptions (pp. 93-107). Houndmills: Palgrave Macmillan.

- Zhao, H. (Ed.). (2008). Zhongyang dianshitai fazhan shi [Historia de CCTV]. Pequín: Zhongguo guang bo dian shi chu ban she [China Radio \& Television Publishing House].

\section{CURRICULUM VITAE. DANI MADRID-MORALES.}

Dani Madrid-Morales es doctorando y profesor asociado del Departamento de Comunicación y Medios de la Universidad de la Ciudad de Hong Kong. Es licenciado en Periodismo y en Estudios de Asia oriental por la Universidad Autónoma de Barcelona y Máster en Relaciones Internacionales por la Universidad Libre de Berlín. Sus áreas de investigación incluyen la comunicación internacional, la estructura de medios en Asia oriental y los flujos comunicativos Sur-Sur. Su investigación ha aparecido en revistas académicas internacionales como Journalism Studies, Journal of Fandom Studies y Communicatio. Es coautor de dos monografías, Historia de Japón y El manga y la animación japonesa, y editor del libro Comunicación y poder en Asia oriental. 